\title{
Ethical Issues in Psychotherapy with Adolescents
}

\author{
Russell D’Souza ${ }^{1}$, Avinash De Sousa ${ }^{2}$ \\ ${ }^{1}$ Director and Head, Asia-Pacific UNESCO Chair in Bioethics, Haifa. \\ ${ }^{2}$ Consultant Psychiatrist and Research Associate, Department of Psychiatry, Lokmanya Tilak \\ Municipal Medical College, Mumbai. \\ Corresponding Author: Russell D'Souza \\ E-mail: russell.f.dsouza@gmail.com
}

Psychotherapy for adolescents with psychological problems has made rapid progress in the last few years. Alongside this progress, there has been the emergence of ethical issues that need to be addressed in various clinical settings. The involvement of parents in adolescent psychotherapy often fuzzes therapeutic boundaries and issues related to confidentiality. The mental health professional working in a hospital or school setting is fraught with multiple problems. The advent of tele-psychotherapy and online psychotherapy has resulted in newer ethical dilemmas for mental health professionals with ethical issues related to communication via that medium. The issues related to parenting, culture and development patterns are also elucidated with a focus on ethical issues for psychotherapy with adolescents [1].

When a psychiatrist / psychotherapist comes face to face with treating an adolescent the aims are in doing what lies in the best interest of the adolescent like protecting the privacy of communication and respecting the adolescent as and the family while promoting and supporting the highest level of development and autonomy for the adolescent in question [2]. The practice of adolescent psychotherapy needs the therapist to achieve and establish rapport with both the adolescent and his/her parents or guardians. Psychotherapy in this special group is a complex and difficult challenge with multiple stakeholders like the child's development in the context of family dynamics, involvement of the school to evaluate the child's educational strengths and weaknesses and sometimes the social work and legal system to look after the needs of the adolescents [3]. We now look at some ethical dilemmas in this domain.

\section{Therapeutic Boundaries and Adolescents}

During any psychotherapy process, subtle instances arise that may pose a risk for boundary problems. Adolescents may challenge boundaries by posing personal questions, such as those about marital status or whether we have children and the career choices that our children have made. Such questions may be purely inquisitive and are potential opportunities to understand the child further. Motivations for these questions can be explored without necessarily having to provide an answer to them. They may also want to connect with us personally via messaging on social media and on various platforms. Therapeutic neutrality can be a helpful guide for making our way with an adolescent in the psychotherapeutic process [4]. Neutrality does not mean that the mental health professional does not care about the child or does not react with feelings to the evolving process. The therapist will neither encourage nor condemn the adolescent or his/her parents and their behavior, but remain interested, wanting to understand their meaning for the adolescent. Mental health professionals have to realize that therapeutic issues with adolescents may be beyond transference and counter-transference and that the adolescents' perceptions of the therapist and vice versa can greatly inform us about the conflicts involved [5].

\section{Autonomy in adolescent psychotherapy}

There is growing recognition of the adolescent to contribute to decisions based on understanding and objectivity. There remain many clinical and ethical indications for adolescents to participate in the decisions about treatment including psychotherapy. The working alliance is strengthened 
when the adolescent feels that he/she has participated in an informed decision to pursue psychotherapy, rather than experience it as imposed on him/her [6]. In today's era where adolescents are quite well read and aware, they must be involved in treatment decisions concerning them when they have the insight and acumen to decide while they have the ability to give assent rather than consent which shall be given by their parents. All decisions in adolescent psychotherapy must be made in the light of parental attitudes, socio-cultural factors, religion and the underlying psychiatric diagnosis and psychopathology [7].

\section{Confidentiality in adolescent psychotherapy}

The psychotherapist dealing with adolescents has a strict responsibility to protect information about the adolescent and his/her family. These principles also serve as the underpinnings of the psychotherapeutic relationship between the adolescent and the mental health professional involved. It is only with the establishment of trust and confidence that a therapeutic space can be created. Within this space, the adolescent can feel sufficiently safe to trust the freedom to reveal all without being judged, retaliated, condemned and reprimanded [8].

In India, more so than the west, we face problems with regard to confidentiality issues when treating adolescents. First, as most of them are accompanied by their parents for consultations and therapy sessions, parents feel that they have a right to know what is going on within the minds of their adolescent may not agree with the confidentiality theory always. Also, many of the times parents pay for the therapy and hence expect the therapist or clinician to listen to them as well as reveal all that transpires within therapy between the therapist and their adolescent.

In school settings, it is even more difficult as a referral to the school counselor without parental approval is often scorned upon by many parents. Professionals conducting psychotherapy with adolescents encounter unique challenges in their efforts to protect their patient's privacy. They rarely operate in a vacuum sealed from interaction with parents and guardians [9].

In Indian society more so than the west, the belief is that a child hides nothing from his parents and that parents must know all when it comes to their child's problems. In fact, it often hurts the parent's ego when the adolescent thinks of going to counselor as parents feel that he has no confidence in them. They believe that he prefers to take problems to an outside party than keep them at home. In the west, psychotherapy for adolescents is better accepted and there are strict laws about confidentiality and almost every issue in psychotherapy, which is lacking in developing countries [10].

In one situation, the child and adolescent psychiatrist may be challenged to protect the privacy of the psychotherapeutic process from parents or guardians who are too intrusive and meddling. In some cases, the therapist may appeal for greater involvement and openness from parents who are perceived as too cold and aloof [11]. Parents and guardians have rights to be informed about any form of treatment conducted for their child, including psychotherapy, and have to be updated on their child's progress. A psychotherapy process with a child or adolescent that is too opaque to the parents may cause dissatisfaction and distrust with the therapist resulting in premature termination of therapy.

Adolescents must be able to trust that he or she has sufficient privacy for the process to be effective. They may not reveal vital information in therapy if they do not perceive this privacy as they may view the therapist as a middle man who just is waiting to reveal information or complain about them to their parents [12]. The psychotherapist has to review with parents the structure of the psychotherapy frame, the type of therapy, interventions in mind and importantly, their rationale. Issues relevant to privacy and confidentiality should be addressed. Parents can be reassured that shall be informed about the process and effects of interventions intermittently. The parents also can be reassured that information suggesting imminent danger to their adolescent or others would not be withheld from them.

\section{Third party communications during adolescent psychotherapy}

There may be multiple requests for information about an adolescent in psychotherapy or treatment progress. This is more so in the school mental health where teachers or the principal may want to know about the adolescent. Sometimes a written report about the same may be demanded. The 
therapist becomes the gatekeeper of information and guardian of the adolescent's privacy. The therapist should respond to any request for release of information with consideration of its appropriateness and necessity and the potential impact of what is released on the adolescent and the psychotherapy process. When considering written or verbal communication to a third party, one should discuss the request with the adolescent and the parents or guardian. Reports requested by schools, courts or hospitals should be reviewed carefully, scrutinizing whether the content is congruent with the needs of the request and whether the reports contain information about the adolescent beyond what is needed. The therapist always should consider the long-term fate of what is released, including whether the setting receiving the material will be able to guard the privacy of these records in ways appropriate for their content [13].

\section{Telepsychotherapy and adolescents}

There are issues which come up with adolescents in that they may quite digitally aware and happy with a telepsychiatry model, while again prescribing medication and monitoring the same gets difficult in these populations. Parents may times want to be around children and adolescents during telepsychiatry counseling sessions and may thus not allow the privacy that is otherwise possible in a clinic setting. Apart from these confidentiality issues, the loss of essential elements of the therapeutic action seen in face to face sessions may be lost compromising the therapist-patient relationship. Talking to children and adolescents via blogs or social networking sites is strictly prohibited for the therapist. The same holds true for chats via messenger or talk networks as the security of these sites itself raises significant concern. It is also prudent that any therapist on a social networking sites refrains from adding the adolescent client as a friend as this involves breach of the therapist-patient relationship. A reliable time and place for contact and therapeutic work is exchanged for telepsychotherapy. Video call is always preferred to audio call to allow face visibility and emotional understanding [14].

\section{Encounters outside the therapeutic space and in public places}

Public encounters with patients pose challenges to privacy and confidentiality and to the adolescent. Although often unpredictable, some public encounters can be anticipated and avoided. When a therapist is aware that an adolescent may be at an event that he or she attends, one may choose to avoid the event or discuss the potential encounter with him or her ahead of time. In general, it is best for therapists to explain to the adolescent and his or her parents that, in the case of encounter outside of the office, one will err in the direction of not acknowledging them unless they initiate an acknowledgment [15].

\section{Counter-transference in adolescent psychotherapy}

Countertransference has been defined as feelings and attitudes toward a patient derived from earlier situations in the analyst's life that have been displaced onto the patient and may include all emotional reactions to the patient, conscious and unconscious, especially those that interfere with understanding and technique. The relevance of counter-transference and neutrality to the ethics of conducting psychotherapy lies in the critical importance of the child and adolescent psychiatrist's attention to his or her emotional reactions to one's patient and one's patient's parents or guardians. Common warning signs in the behavior of the therapist include recurrent lateness to sessions, extensions of sessions, touching of patients, gifts to the patient, and contact with the patient outside of scheduled sessions, especially outside of the office setting [16].

\section{Conclusions}

As noted above there are many ethical issues governing the conduct of psychotherapy with adolescents. Advances in development, globalization, the technology age, variations in parenting, religion, cultural factors, social and economic factors, legal requirements, the therapy setting and the therapist's own judgment should stimulate consideration of how these ethical principles apply to various situations. However, the most pivotal remains the psychotherapist's obligation to create and protect the integrity of the psychotherapeutic space to provide the adolescent the freedom to identify, examine, explore, and hopefully resolve the issues that bring one to treatment. 


\section{REFERENCES}

1. Dell ML, Kinlaw K. Theory can be relevant: An overview of bioethics for the practicing child and adolescent psychiatrist. Child Adolesc Psychiatr Clin N Am 2008;17(1): 1-19.

2. Schetky DH. Boundaries in child and adolescent psychiatry. Child Adolesc Psychiatr Clin N Am 1995;4(4):769-78.

3. Simon RI, Williams IC. Maintaining treatment boundaries in small communities and rural areas. Psychiatr Serv 1999;50(11):1440-6.

4. Fuentes J, Martin-Arribas MC. Bioethical issues in neuropsychiatric genetic disorders. Child Adolesc Psychiatr Clin N Am 2007;16(3):649-61.

5. Stalets MM, Luby JL. Preschool Depression. Child Adolesc Psychiatr Clin N Am 2006;15(4): 899917.

6. DeSocio JE. Assessing self development through narrative approaches in child and adolescent psychotherapy. J Child Adolesc Psychiatr Nurs 2005;18(2):53-61.

7. March JS. The future of psychotherapy for mentally ill children and adolescents. J Child Psychol Psychiatry 2009;50(1-2):170-9.

8. Henretty JR, Levitt HM. The role of therapist self disclosure in psychotherapy : a qualitative review. Clin Psychol Rev 2010;30(1):63-77.

9. Koocher GP. Ethical issues in psychotherapy with children and adolescents. J Clin Psychol 2003; 59(11):1247-56.

10. Josephson AM. Formulation and treatment: integrating religion and spirituality in clinical practice. Child Adolesc Psychiatr Clin N Am 2004;13(1):71-84.

11. Nigg JT. Temperament and developmental psychopathology. J Child Psychol Psychiatry 2006; 47(3-4):395-422.

12. Recupero PR. Ethics of medical records and professional communications. Child Adolesc Psychiatr Clin N Am 2008; 17:37-51.

13. Recupero PR. E-mail and the psychiatrist patient relationship. J Am Acad Psychiatry Law 2005; 33:465-75.

14. Ascherman LI, Rubin S. Current ethical issues in child and adolescent psychotherapy. Child Adolesc Psychiatr Clin N Am 2008;17(1):21-35.

15. Welfel ER. Ethics in counseling \& psychotherapy. Cengage Learning; 2015.

16. Koelch M, Fegert JM. Ethics in child and adolescent psychiatric care: An international perspective. Int Rev Psychiatry 2010;22(3):258-66.

Acknowledgements - Nil

Source of Funding - Nil

Conflict of Interest - Nil 